Association for Information Systems AIS Electronic Library (AISeL)

Wirtschaftsinformatik Proceedings 1999

Wirtschaftsinformatik

February 1999

\title{
Die Substitution von Dienstleistungen durch Informationsprodukte auf elektronischen Märkten
}

Florian Bieberbach

Universität TU München, bieberbach@aib.wiso.tu-muenchen.de

Michael Herrmann

Universität TU München, hermann@aib.wiso.tu-muenchen.de

Follow this and additional works at: http://aisel.aisnet.org/wi1999

\section{Recommended Citation}

Bieberbach, Florian and Herrmann, Michael, "Die Substitution von Dienstleistungen durch Informationsprodukte auf elektronischen Märkten" (1999). Wirtschaftsinformatik Proceedings 1999. 5.

http://aisel.aisnet.org/wi1999/5

This material is brought to you by the Wirtschaftsinformatik at AIS Electronic Library (AISeL). It has been accepted for inclusion in Wirtschaftsinformatik Proceedings 1999 by an authorized administrator of AIS Electronic Library (AISeL). For more information, please contact elibrary@aisnet.org. 


\section{Die Substitution von Dienstleistungen durch Informationsprodukte auf elektronischen Märkten}

Florian Bieberbach

Universität TU München (bieberbach@aib.wiso.tu-muenchen.de)

Michael Hermann

Universität TU München (hermann@aib.wiso.tu-muenchen.de)

\section{Inhalt}

1 Dienstleistungen im Umbruch

2 Dienstleistungen und Informationsgüter

3 Elektronische Märkte im Internet

4 Elektronische Märkte als Katalysator für die Substitution von Informationsdienstleistungen durch Informationsprodukte

5 Implikationen für Dienstleistungsanbieter und die Rolle der Wirtschaftsinformatik 


\begin{abstract}
In der öffentlichen Diskussion werden mit den Begriffen "Dienstleistungsgesellschaft" und "Informationsgesellschaft" zukünftige Entwicklungspfade moderner Volkswirtschaften umrissen. Die industrielle Produktion materieller Güter verliert an Bedeutung, was Wertschöpfung und Beschäftigung betrifft. An ihre Stelle treten Dienstleistungen - und vor allem die Produktion von Informationen. Diese Entwicklung wird im wesentlichen bestimmt durch den rasanten technischen Fortschritt in den Informations- und Kommunikationstechnologien (IuK-Technologien). Durch den Einfluß dieser Technologien steigt aber nicht nur die quantitative Bedeutung des Wertes von Informationen an, sie beeinflussen darüber hinaus massiv die Bedingungen, unter denen Informationen als Güter produziert und gehandelt werden. Eine Unterscheidung von Informationsgütern in Informationsprodukte und Informationsdienstleistungen ermöglicht einen genaueren Einblick in diese Veränderungen.
\end{abstract}

Während die Produktion von Informationsdienstleistungen immer die Einbeziehung externer Produktionsfaktoren des Nachfragers in die Leistungserstellung voraussetzt, sind Informationsprodukte dadurch charakterisiert, daß sie industriell, d.h. massenhaft und für einen anonymen Markt, produziert werden können. Während bei der Erstellung von Informationsprodukten prinzipiell die gleichen Produktivitätsfortschritte wie bei der industriellen Fertigung von Sachgütern realisierbar sind, gelten für die Produktion von Informationsdienstleistungen aufgrund der Bedeutung des externen Faktors die gleichen Beschränkungen wie für die Produzenten aller Dienstleistungen.

Die Vorteile, die durch die massenhafte industrielle Fertigung von Sachgütern in der Vergangenheit erzielt werden konnten, lassen sich auch bei der Produktion von Informationsprodukten erzielen, wenn immer mehr bestehende Informationsdienstleistungen durch Informationsprodukte substituiert werden. Von besonderer Bedeutung ist für diesen Substitutionsprozeß die Herausbildung elektronischer Märkte auf Basis des weltumspannenden Internet. Aufgrund der spezifischen Charakteristika elektronischer Märkte sind Informationsdienstleistungen der Möglichkeit zur Substitution durch Informationsprodukte in verstärktem Maße ausgesetzt. Die Fähigkeit, aus bestehenden Informationsdienstleistungen Informationsprodukte zu entwickeln wird zu einem kritischen Erfolgsfaktor für Anbieter von Informationsgütern. Gleichzeitig eröffnet diese Entwicklung Potentiale für die Entwicklung innovativer Informationsdienstleistungen. Die Bedeutung von Informationen als wirtschaftliches Gut wird durch beide Entwicklungen in der Zukunft noch weiter zunehmen. Der Weg, der in die "Informationsgesellschaft" führt, ist also nicht notwendigerweise auch ein Weg in die "Dienstleistungsgesellschaft". 


\section{Dienstleistungen im Umbruch}

Nach den Zahlen des Online Banking Report nutzen derzeit rund fünf Millionen US-Bürger eine Form von Online-Banking. Bis Ende 2001 wird diese Zahl auf 22 Millionen steigen; das entspricht 21\% der Bevölkerung der USA (Wall Street Journal, 19.5.98). Die Umsätze bei Online-Finanzgeschäften werden nach Schätzungen von Forrester Research bis 2001 auf fünf Milliarden US-Dollar steigen. Auch in Deutschland entscheiden sich mehr und mehr Kunden für die neue Form, Bankgeschäfte $\mathrm{zu}$ erledigen. Daß viele Online-Broker bewußt keinerlei Kundenberatung bieten, scheint für diese Kunden nicht mehr wichtig zu sein.

Ähnliche Entwicklungen stehen auch anderen Dienstleistungsbereichen bevor: in der Reisebranche erwartet Forrester bis 2001 Online-Umsätze von über 7 Milliarden US-Dollar. Dies stellt die klassischen Reisebüros vor große Herausforderungen und wird den ohnehin schon sehr intensiven Wettbewerb in diesem Markt noch verschärfen. Kleineren Brachen wie Übersetzungs- und Schreibbüros droht die weitgehende Substitution durch Diktier- und Übersetzungssoftware. Lernsoftware ersetzt zum Teil den Unterricht, Telemedizin den Arztbesuch.

Diese Beispiele ließen sich lange fortsetzen. In den 80er Jahren erlebte der produzierende Sektor in Deutschland durch den zunehmenden Einsatz von Informationstechnologie (IT) massive Umstrukturierungen und Rationalisierungen. Eine ähnliche Entwicklung wurde für die 90er Jahre dem Dienstleistungssektor prophezeit. Was die Anbieter von Dienstleistungen, insbesondere informationsintensiven Dienstleistungen jedoch erleben, geht über Rationalisierung hinaus. Hier müssen ganze Unternehmen und Berufsgruppen befürchten, komplett zu verschwinden.

Dies steht scheinbar im Gegensatz zu den Prognosen, die in Deutschland einen starken Anstieg des Beschäftigtenanteils im Bereich der Informationsdienstleistungen vorhersagen (vgl. Abbildung 1). Es stellt sich die Frage, ob der Weg in die Informationsgesellschaft tatsächlich gleichzeitig der Weg in die (Informations-) Dienstleistungsgesellschaft ist, oder ob uns nicht vielleicht sogar ein neuer Anstieg des produzierenden Sektors bevorsteht, mit dem Unterschied, $\mathrm{da}$ in diesem Sektor jetzt auch Informationsgüter industriell, d.h. massenhaft und für einen anonymen Markt, produziert werden.

1 Kraus z.B. schätzt, daß über 6 Mio. Arbeitsplätze im Dienstleistungssektor in Deutschland (nach Abgrenzung des Statistischen Bundesamtes) durch den Einsatz von IT wegfallen könnten (vgl. Kraus 1997, S. 125). In seiner Schätzung berücksichtigt er ausdrücklich nicht die durch den Einsatz der Technologie neu entstehenden Arbeitsplätze. 


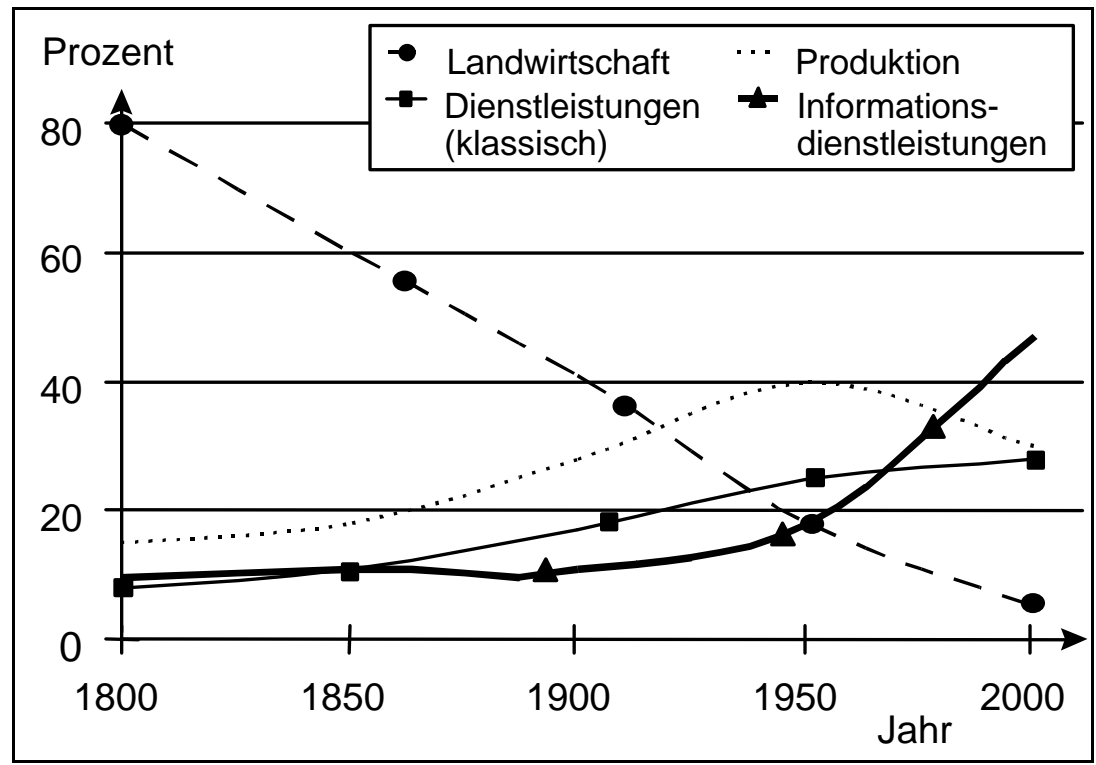

Abbildung 1: Prognostizierte Verschiebung der Beschäftigung durch sektoralen Strukturwandel (Gries 1995)

Von besonderem Interesse sind in diesem Zusammenhang die Auswirkungen elektronischer Märkte, die mit der rasanten Diffusion des Internet immer stärker auch für marktliche Beziehungen zwischen Produzenten und Konsumenten auf Massenmärkten an Bedeutung gewinnen. Es werden die Charakteristika elektronischer Märkte analysiert, um darauf aufbauend Aussagen über Veränderungen der marktgerichteten Produktion von Informationen ableiten zu können. Anschließend werden die Konsequenzen aus diesen für die Akteure auf den sich wandelnden Märkten diskutiert.

\section{Dienstleistungen und Informationsgüter}

Im folgenden Abschnitt werden wesentliche Eigenschaften von Dienstleistungen und Informationsgütern diskutiert, um Gemeinsamkeiten zu identifizieren und Unterschiede zu verdeutlichen. Die Differenzierungskriterien sind hilfreich, um die Auswirkungen der Elektronisierung von Marktprozessen auf das Verhältnis von Dienstleistungen und Informationsgütern präzise beschreiben zu können.

Der Dienstleistungssektor nimmt in den entwickelten Volkswirtschaften einen immer größer werdenden Anteil an der gesamten Wertschöpfung und Beschäftigung ein. So betrug in der Bundesrepublik Deutschland 1997 der Anteil der Wertschöpfung der Dienstleistungsunternehmen nach der sektoralen Abgrenzung der Volkswirtschaftlichen Gesamtrechnung 63,5\%, der Anteil der Erwerbstätigen 
in diesem Sektor $62,8 \%^{2}$ (Statistisches Bundesamt 1998, http://www.statistikbund.de/). Diese Zahlen markieren den gegenwärtigen Stand des Tertiarisierungsprozesses in der deutschen Volkswirtschaft, der mit großer Wahrscheinlichkeit noch weiter voranschreiten wird.

Der klassische theoretische Erklärungsansatz für die Verschiebung der Beschäftigung in den tertiären Sektor ist die sogenannte "Drei-Sektoren-Hypothese", die von Colin Clark (1940) und Jean Fourastié (1949) (vgl. Reichwald/Möslein 1995, S. 331) entwickelt wurde. Nach dieser Hypothese ist die Verschiebung der Beschäftigung von der Agrarproduktion (primärer Sektor) über die Industrieproduktion (sekundärer Sektor) hin zu der Dienstleistungsproduktion ein nicht umkehrbarer, stetig voranschreitender Prozeß in der Entwicklung von Volkswirtschaften.

Die Erklärung für diese zwangsläufige Entwicklung ist in der Abgrenzung des tertiären Sektors der Begründer der Drei-Sektoren-Hypothese zu finden (vgl. Meyer 1983, S. 8): Nach Fourastié ist ein konstitutives Merkmal des tertiären Sektors, daß dort technischer Fortschritt nur im geringem Umfang möglich sei. Demzufolge seien Steigerungen der Arbeitsproduktivität bei Dienstleistungstätigkeiten ebenfalls nur begrenzt realisierbar - ganz im Gegensatz zum sekundären Sektor, in dem der technische Fortschritt sich sehr stark auswirkte und immense Produktivitätssprünge ermöglichte.

Die Abgrenzung von Dienstleistungen über die generelle Abwesenheit technischen Fortschritts und die damit einhergehende Stagnation in der Produktivitätsentwicklung wurde als unbefriedigend empfunden. Es wurden daher andere Kriterien entwickelt, die konstitutive Merkmale herausstellen sollten. ${ }^{3}$ Eine einheitliche Definition von Dienstleistungen existiert zwar bis heute nicht, aber ein Kriterium, das von allen Autoren als wesentlich für Dienstleistungen gesehen wird, ist der Einsatz eines externen Produktionsfaktors bei der Leistungserstellung. (vgl. Maleri 1994, S. 39; Bode 1997, S. 462; Meffert/Bruhn 1998, S. 32; Corsten 1985, S. 134). Darunter werden Produktionsfaktoren verstanden, die durch den Leistungsnehmer in den Produktionsprozeß eingebracht werden und nicht vollständig vom Dienstleistungsproduzenten kontrolliert werden können. Dabei kann es sich z.B. um Informationen handeln (im Falle der Unternehmensberatung), um Objekte im Besitz des Leistungsnehmers (z.B. Autoreparatur) oder um den Leistungsnehmer selbst (z.B. bei einer ärztlichen Untersuchung). Aus der Existenz des externen Faktors folgt, daß

- eine direkte Interaktion zwischen Anbieter und Nachfrager stattfinden muß, in der die internen Produktionsfaktoren (des Anbieters) mit den externen (des Nachfragers) kombiniert werden (vgl. Maleri 1994, S. 130), die in vielen

2 Bei einer Betrachtung der Tätigkeiten der Erwerbspersonen erhöht sich dieser Anteil für die Bundesrepublik Deutschland in 1997 sogar auf 73,8\% (vgl. Haisken-De New, Horn, Schupp und Wagner 1998).

3 Zur Diskussion um den Dienstleistungsbegriff vgl. z.B. Corsten (1990), S. 17 ff. und Maleri (1994), S. 2 ff. 
Fällen einen zeitgleichen Kontakt zwischen Anbieter und Nachfrager erforderlich machen (ebd., S. 87),

- eine Standardisierung der Leistung nur im begrenzten Umfang möglich ist ${ }^{4}$ da die externen Faktoren mit dem jeweiligen Nachfrager variieren können.

Umstritten ist dagegen, wie das Merkmal der Immaterialität im Kontext der Dienstleistungen zu bewerten ist (vgl. Maleri 1994, S. 81; Corsten 1985, S. 90). Diese Frage ist auch hier von zentraler Bedeutung, da die Stellung von Informationsgütern in Bezug zu den Dienstleistungen untersucht wird. Sind Informationsgüter automatisch auch Dienstleistungen, da sie wie diese das Merkmal der Immaterialität aufweisen $?^{5}$ Oder begründen sie neben den Dienstleistung eine eigene Kategorie unter den immateriellen Gütern? ${ }^{6}$ Das Merkmal der Immaterialität eignet sich natürlich insbesondere zur Abgrenzung der Dienstleistungen von den materiellen Sachgütern, für die Abgrenzung zu den Informationsgütern ist dagegen die Bedeutung des externen Faktors hilfreicher.

Nach Bode sind Informationen dann Wirtschaftsgüter, wenn sie "zweckgeeignet, verfügbar, übertragbar und knapp sind sowie auf wirksame Marktnachfrage treffen." (vgl. zu den folgenden Ausführungen Bode 1997, S. 461 ff.) ${ }^{7}$. Zieht man zur Abgrenzung der (immateriellen) Informationsgüter von den (immateriellen) Dienstleistungen die Bedeutung des externen Faktors heran, so lassen sich Informationsgüter in Informationsprodukte und Informationsdienstleistungen unterscheiden. Informationsdienstleistungen sind dadurch charakterisiert, daß der Nachfrager bei der Leistungserstellung wesentlichen Input als externen Produktionsfaktor einbringt (i.d.R. werden dies wiederum Informationen sein, wie z.B. bei der Steuerberatung). Informationsprodukte können dagegen vom Produzenten autonom ohne Interaktion mit dem Nachfrager erstellt werden (wie z.B. Bücher, Filme, Fernsehsendungen, Musikaufnahmen). ${ }^{8}$

4 Die begrenzte Möglichkeit zur Standardisierung, und somit zur Realisierung von Produktivitätssteigerungen, ist hier nicht konstitutives Merkmal der Dienstleistungen, sondern folgt aus der Bedeutung des externen Faktors. Maleri hält dagegen auch Dienstleistungen generell für standardisierbar (vgl. Maleri 1994, S. 53), allerdings tritt dann die Bedeutung des externen Faktors in den Hintergrund.

5 So z.B. in der Gütersystematik nach Corsten (1990, S. 17).

6 Wie in der Gütersystematik von Maleri (1994, S. 50) oder von Meffert/Bruhn (1997, S. 28).

7 Informationen allgemein - also unabhängig von ihrer wirtschaftlichen Verwertbarkeit werden von Bode als "Wissensbestandteile, die in Form menschlicher Sprache repräsentiert sind" definiert. (Bode 1997, S. 459).

8 Eine einheitliche Zuordnung von Computersoftware in diese Kategorien ist nicht möglich: Je nachdem, ob der Nachfrager in die Leistungserstellung eingebunden ist (wie bei Individualsoftware) oder ob dies nicht der Fall ist (wie bei Standardsoftware), wird die jeweilige Leistung in eine der beiden Kategorien eingeordnet. 
Mit der Unterscheidung in Informationsprodukte und Informationsdienstleistungen kann auch die Frage nach Standardisierung von Informationsgütern und den damit möglichen Produktivitätsgewinnen verdeutlicht werden: Während bei der Erstellung von Informationsprodukten prinzipiell die gleichen Produktivitätsfortschritte wie bei der industriellen Fertigung von Sachgütern realisierbar sind, gelten für die Produktion von Informationsdienstleistungen aufgrund der Bedeutung des externen Faktors die gleichen Beschränkungen, wie für die Produzenten anderer Dienstleistungen.

Damit wird durch die Fokussierung der Betrachtung auf den externen Faktor eine Analogie zu der "klassischen" Unterteilung in materielle Güter und Dienstleistungen hergestellt: Entscheidend ist letztendlich der Einsatz einer Produktionstechnologie, die eine vom Nachfrager unabhängige Massenproduktion und die damit erzielbaren economies of scale ermöglicht, dies gilt für Informationsprodukte wie für Sachgüter.

Im Hinblick auf elektronische Märkte unterscheiden Choi/Stahl/Whinston digitale Produkte ${ }^{9}$ anhand der Art der Übertragung in gelieferte und interaktive Produkte (vgl. Choi/Stahl/Whinston 1997, S. 76). Interaktive Produkte sind dabei durch Echtzeit-Anwendungen und durch die Interaktion in sukzessiven Anfragen und Antworten charakterisiert. Beispiele hierfür sind Spiele oder die Konsultierung eines Arztes. Gelieferte digitale Produkte benötigen diese Interaktion nicht: Nachdem die Informationen geliefert wurden, findet keine Interaktion mehr statt. Der Liefervorgang selbst kann dabei auch automatisiert werden. Dabei weisen Whinston et al. auch darauf hin, daß viele Produkte, die über das Internet geliefert werden, im allgemeinen Sprachgebrauch als interaktiv bezeichnet werden, obwohl keine echte Interaktion zwischen Anbieter und Nachfrager stattfindet (Choi/Stahl/Whinston 1997, S. 77). Wie bei Bode ist auch in dieser Klassifizierung die Einbeziehung des Nachfragers bei der Leistungserstellung das wichtigste Diskriminierungskriterium.

Im folgenden wird gezeigt, daß auf elektronischen Märkten nicht nur beide Arten von Informationsgütern gehandelt werden können, sondern daß durch die speziellen Charakteristika der elektronischen Märkte selbst ein Trend zur Substitution von bestehenden Informationsdienstleistungen durch Informationsprodukte begünstigt wird. Im folgenden Abschnitt werden daher zunächst die Charakteristika elektronischer Märkte analysiert.

9 Über digitale Produkte sagen Choi/Stahl/Whinston: "Alles, was man über das Internet senden und empfangen kann, ist ein potentielles digitales Produkt." (vgl. Choi/Stahl/Whinston 1997, S. 62). Dabei ist "Information ein primäres Beispiel für ein digitales Produkt" (ebd., S. 61). 


\section{Elektronische Märkte im Internet}

Das weltumspannende Internet beeindruckt seit Jahren durch sein enormes Wachstum. Auch wenn Benutzerzahlen schwierig abzuschätzen sind, prognostiziert der Economist, daß bis zum Jahr 2000 schon 10\% der Weltbevölkerung das Internet nutzen werden (Anderson 1997). Als neues Medium hat es die Informations- und Kommunikationsgewohnheiten vieler Menschen bereits stark verändert, und es ist absehbar, daß auch große Bereiche der Wirtschaft weitreichenden Veränderungen unterworfen sein werden. Das Internet kristallisiert sich als die dominante Handelsplattform der Zukunft heraus.

Insbesondere für Dienstleistungsunternehmen stellt sich die Frage, welche Auswirkungen die zunehmende Bedeutung des Internet-Handels auf Leistungsspektrum und Unternehmensstrategien haben werden. Dies soll im folgenden näher untersucht werden. Der Fokus liegt dabei auf marktlich gehandelten Dienstleistungen; auf unternehmensinterne Dienstleistungen, insbesondere in verteilten Arbeits- und Organisationsformen, lassen sich jedoch einige Ergebnisse übertragen.

Im Bereich elektronischer Märkte haben sich sowohl in der deutsch- als auch in der englischsprachigen Literatur noch keine klaren Definitionen durchgesetzt. So werden die Begriffe elektronischer Markt (electronic market), elektronischer Marktplatz (electronic marketplace, marketspace, virtual market) häufig ohne klare Abgrenzungen oder gar synonym gebraucht (vgl. z.B. Bakos 1998; Choi/Stahl/Whinston 1997; Rayport/Sviokla 1994; Ware/Gebauer/Hartmann/ Roldan 1997; Benjamin/Wigand 1995). Es scheint daher zunächst angebracht, die Verwendung der Begriffe in diesem Artikel kurz zu erläutern.

Elektronische Märkte werden in der deutschsprachigen Literatur meist nach Schmid (1993) definiert als "informationstechnische Systeme zur Unterstützung aller oder einzelner Phasen und Funktionen der marktmäßig organisierten Leistungskoordination“. Anzumerken ist jedoch, daß das Verständnis von elektronischen Märkten als "informationstechnischen Systemen" im Widerspruch steht zu den in der Betriebs- und Volkswirtschaftslehre üblichen Definitionen eines Marktes. In der Betriebswirtschaftslehre wird unter einem Markt meist ein "ökonomischer Ort verstanden, auf dem Güterangebot und -nachfrage zusammentreffen und der damit Tauschvorgänge ermöglicht" (Picot/Reichwald/ Wigand 1998, S. 25).

In der Volkswirtschaftslehre werden Märkte durch Güter definiert (z.B. der Markt für Motorräder oder Vermögensberatung) und nach der Substituierbarkeit durch andere Güter bzw. die Kreuzpreiselastizität abgegrenzt. "Er [der Markt] umfaßt entweder ein homogenes Gut oder eine Gruppe verschiedener Produkte, die für mindestens ein Gut der jeweiligen Gruppe enge Substitute (oder Komplemente) sind" (Tirole 1995, S. 27). 
Nach diesen Vorstellungen sind elektronische Märkte Teilmärkte bestimmter Produkt- und Dienstleistungsmärkte, die sich dadurch abgrenzen, daß zumindest einige Phasen der Markttransaktionen über Informations- und Kommunikationssysteme abgewickelt werden. Für den elektronischen Markt als abstrakten Ort des Austausches sind die zugrundeliegenden, technischen Systeme eine notwendige, nicht jedoch hinreichende Bedingung.

Durch die Entwicklungen im Bereich des Internet kristallisieren sich verschiedene Funktionen im Zusammenhang mit elektronischen Märkten heraus, die in Abbildung 2 veranschaulicht werden:

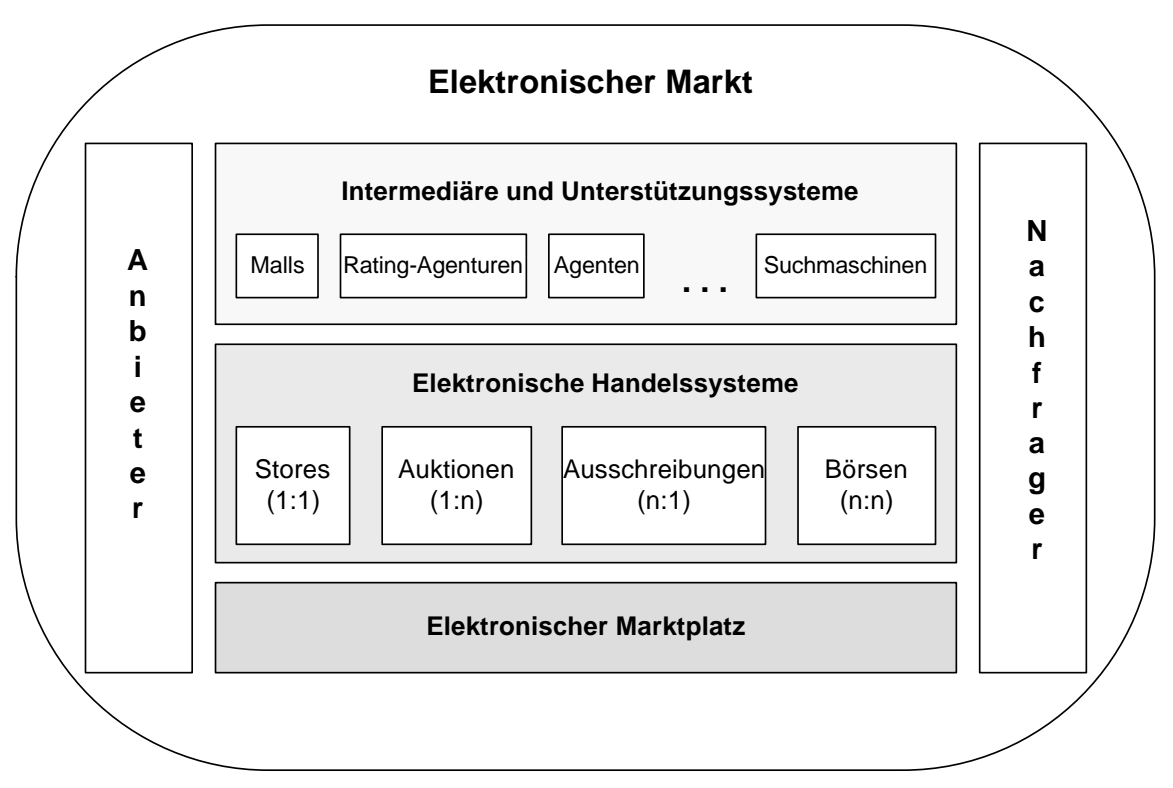

Abbildung 2: Schichtenmodell elektronischer Märkte

Die unterste Ebene bilden die elektronischen Marktplätze. Elektronische Marktplätze sind Informations- und Kommunikationsinfrastrukturen, die geeignet sind, als Basis marktmäßig organisierter Leistungskoordination zu dienen. Beispielsweise kann die Internet-Infrastruktur mit den zugehörigen Standards (TCP/IP etc.) und Diensten (WWW etc.) als elektronischer Marktplatz angesehen werden. Weitere Beispiele sind Online-Dienste, Minitel oder auch ein Fernsehkanal in Verbindung mit einem Rückkanal (z.B. Home Order Television und Telefon). Eine solche Unterscheidung zwischen Markt und Marktplatz entspricht der traditionellen Unterscheidung in der angelsächsischen Literatur zwischen market und marketplace, "also dem Markt als abstraktem Gebilde und jenem im geographischen Sinne" (Nieschlag/Dichtl/Hörschgen 1994, S. 92). Elektronische Marktplätze ersetzen geographisch abgegrenzte. 
Auf der Basis eines elektronischen Marktplatzes können nun Anbieter und Nachfrager ihre Handelsbereitschaft signalisieren und in marktliche Austauschprozesse treten. Elektronische Handelssysteme sind Informations- und Kommunikationssysteme, die speziell der Koordination und Abwicklung marktlicher Leistungsaustausche auf elektronischen Marktplätzen dienen. Sie sollen hier grob nach der Anzahl der Anbieter und Nachfrager in vier Gruppen unterteilt werden:

Stores sind Systeme, die entweder von einem Anbieter oder von einem Nachfrager betrieben werden und in denen jeweils ein Anbieter mit einem Nachfrager in Beziehung tritt und ggf. nach Verhandlungen ein Vertrag sowie ein Leistungsaustausch zustande kommen (vgl. Choi/Stahl/Whinston 1997, S. 253). Meist ist es die Anbieterseite, die mit solchen Stores auf dem elektronischen Marktplatz auftritt. Beispiele sind elektronische Kataloge oder übliche kommerzielle Sites im WWW.

Bei Auktionssystemen treten mehrere Nachfrager zueinander in Wettbewerb um die Leistung eines Anbieters. Im allgemeinen erhält derjenige den Zuschlag, der den höchsten Preis zu zahlen bereit ist. Elektronische Ausschreibungen sind dazu spiegelbildlich: hier spezifiziert ein Nachfrager eine Leistung, um deren Erbringung mehrere Anbieter konkurrieren.

Der Markt entsteht in diesen ersten drei Fällen erst durch den Wettbewerb verschiedener Stores, Auktionen oder Ausschreibungen. Können Käufer auf der Suche nach einem Buch zwischen verschiedenen Stores, in denen Bücher angeboten werden, wählen, so kann man von einem elektronischen Markt für Bücher sprechen.

Nur bei elektronischen Börsen wird der Markt weitgehend in einem einzelnen IuK-System abgebildet. Die dort entstehenden elektronischen Märkte kommen in ihrer Funktionsweise dem Ideal eines vollkommenen Marktes am nächsten. Hier treffen viele Anbieter auf viele Nachfrager, und über definierte Mechanismen wird ein gemeinsamer, im allgemeinen umsatzmaximierender, Preis festgelegt.

Die auf der Schicht der Handelssysteme aufsetzenden Intermediäre sind nach Choi/Stahl/Whinston (1997, S. 375) definiert als „Mittler, die Transaktionen zwischen potentiellen Handelspartnern erleichtern“. Die sich herauskristallisierenden Funktionen von Intermediären auf elektronischen Märkten liegen insbesondere im Bereich der Informationsunterstützung bei Such- und Entscheidungsvorgängen. So fassen beispielsweise Malls verschiedene Stores unter einem gemeinsamen „virtuellen Dach“ zusammen und treffen damit eine Vorauswahl für die Nachfrager bzw. eine gemeinsame Marketing-Plattform für die Anbieter. Rating-Agenturen bewerten Anbieter und Leistungen im Netz und erleichtern damit ihren Kunden das Finden eines geeigneten Handelspartners. Erste Ansätze mit intelligenten Agenten und automatisierten Verhandlungen zeigen, daß sich hier noch ein weites Feld für innovative und zunehmend spezialisierte Unterstützungsleistungen bietet. 
Die Ökonomik elektronischer Märkte ist derzeit Gegenstand zahlreicher wissenschaftlicher Untersuchungen. Im folgenden sollen einige Aspekte herausgegriffen werden, die insbesondere für den Dienstleistungsbereich von Bedeutung sind.

\section{Elektronische Märkte als Katalysator für die Substitution von Informationsdienstleistungen durch Informationsprodukte}

Elektronische Märkte beschleunigen die Substitution von Informationsdienstleistungen durch Informationsprodukte. Dieser Substitutionsprozeß findet auf verschiedenen Ebenen statt. Zum einen können mit dem Handel verbundene Dienstleistungen selbst Gegenstand eines Substitutionsprozesses werden. Mit elektronischen Handels- und Unterstützungssystemen wird die Informationsfunktion des Marktes, die auf nicht-elektronischen Märkten von Händlern, Maklern und/oder den Marktteilnehmern als Dienstleistung erbracht wird, durch Informationsprodukte realisiert. ${ }^{10}$

Der Fortschritt in der Entwicklung der IuK-Technologie erlaubt zum anderen die Umwandlung immer komplexerer Informationsdienstleistungen (z.B. Beratungsdienstleistungen) in Informationsprodukte. Diese stellen in digitalisierter Form die ideale Ware für elektronische Märkte dar, da für sie die Transportkosten auf elektronischen Marktplätzen drastisch zurückgehen. Elektronische Märkte erhöhen somit die relativen Kostenvorteile solcher Informationsprodukte.

Abbildung 3 zeigt einige Beispiele für Informationsdienstleistungen, deren Substitution durch Informationsprodukte durch elektronische Märkte begünstigt wird. Es handelt sich i.d.R. um standardisierte Leistungen, die durch IuKSysteme automatisiert erbracht werden können. Der technische Fortschritt in der Entwicklung von Computerhardware und Computersoftware verschiebt jedoch die Grenze der Automatisierbarkeit immer weiter, so daß immer komplexere Leistungen in Computersystemen abgebildet werden können.

Die Substitution von Informationsdienstleistungen durch Informationsprodukte fand und findet natürlich auch außerhalb elektronischer Märkte statt. In Abbildung 3 sind zur Veranschaulichung dafür weitere Beispiele für diesen Substitutionsprozeß aufgeführt.

10 Malone, Yates, Benjamin sprechen in diesem Zusammenhang vom Brokereffekt elektronischer Märkte (vgl. Malone/Yates/Benjamin 1986, S. 9). Die Informationsprodukte können den Marktteilnehmern natürlich auch von einer dritten Partei zur Nutzung überlassen werden. In diesem Fall kann man wieder von einer Dienstleistung sprechen (jedoch nicht von einer Informationsdienstleistung). 


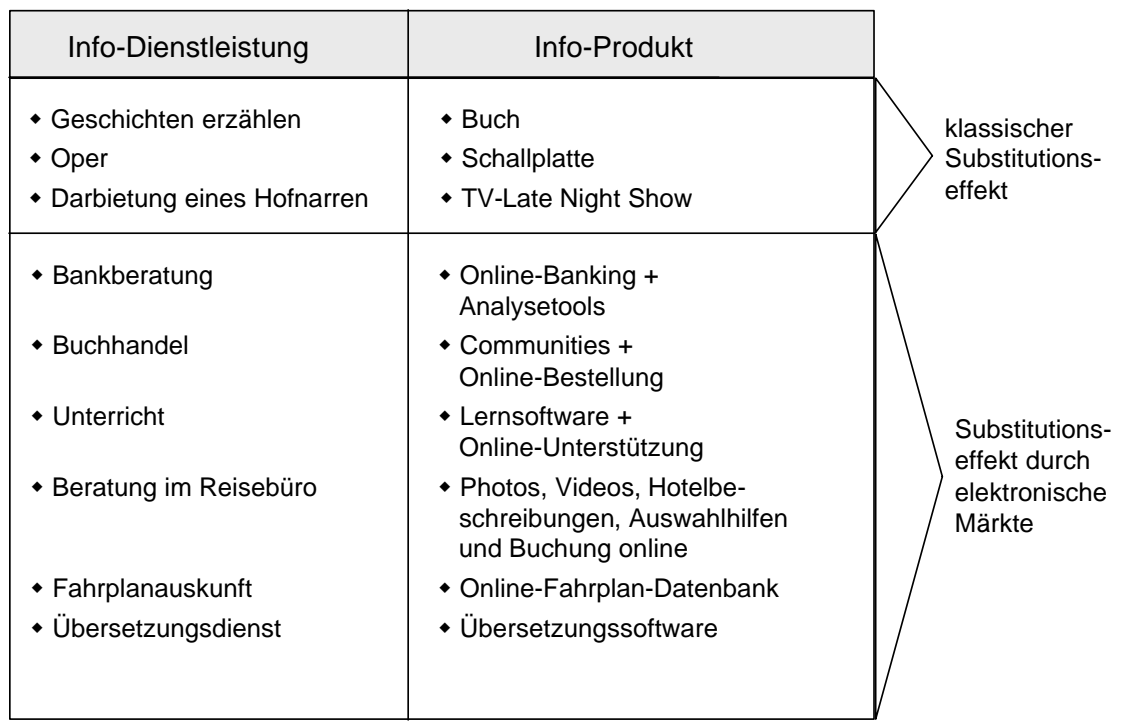

\section{Abbildung 3: Beispiele für die Substitution von Informationsdienstleistungen durch Informationsprodukte}

Der Substitutionseffekt zieht weitreichende ökonomische Konsequenzen für die Erstellung der betreffenden Informationsgüter nach sich. Einer der nächstliegenden Effekte elektronischer Märkte ist die Verbilligung des Vertriebs von Informationsgütern durch das kontinuierliche Sinken der Kosten der Informationsübertragung. Dies hat eine stark abnehmende Bedeutung regionaler Grenzen für den Vertrieb von Informationsgütern zur Folge. Die direkte Einbeziehung des Kunden bei der Erstellung der Informationsdienstleistungen ist hier nur noch über Telekommunikationsmedien, d.h. definierte technische Schnittstellen, möglich.

Neben den regionalen verlieren aber zeitliche Grenzen ebenso ihre Bedeutung. Im Internet sind 24-Stunden-Öffnungszeiten völlig selbstverständlich, während die Erbringung entsprechender Leistungen durch menschliche Akteure z.B. in internationalen Call-Centern dagegen auf große Probleme bei der Arbeitsorganisation stößt. Der durch Rechner erbrachte, automatisierte Vertrieb von Informationsprodukten ist hier klar im Vorteil.

Die Auflösung von Marktgrenzen führt außerdem zu einer räumlichen und zeitlichen Ausdehnung der Absatzreichweite einzelner Unternehmen und damit zu einem Verschmelzen vieler bisher getrennter Teilmärkte. In diesen größeren Märkten sind Kostenführerschaftsstrategien über economies of scale einfacher zu realisieren, was ebenfalls der Massenfertigung von Informationsprodukten gegenüber den Dienstleistungen einen Vorteil verschafft.

Häufig reagieren Unternehmen auf den verschärften Wettbewerb durch Differenzierung und Kundenorientierung (ECE 1998, S. 22). Dies über ein personalinten- 
sives Dienstleistungsangebot zu realisieren, scheint jedoch eine gewagte Strategie, angesichts der Tatsache, $\mathrm{da} ß$ es im Internet vergleichsweise einfach möglich ist, automatisiert kundenindividuelle Angebote (mass customization) bereitzustellen (vgl. Bakos 1998). ${ }^{11}$

Profitieren können von diesem verschärften Wettbewerbsbedingungen in erster Linie die Nachfrager der Informationsgüter. Die automatisierte Erstellung der Leistungen führt zu Kostensenkungspotentialen, die bei funktionierendem Wettbewerb zu Preissenkungen für die Informationsgüter führen. ${ }^{12}$

Gleichzeitig eröffnen sich Potentiale für die Entwicklung innovativer Informationsdienstleistungen, die sich durch die Entbindung der Dienstleistungsproduzenten von den Standardleistungen ergeben. Eine wesentliche Voraussetzung für die Realisierung dieser Potentiale ist eine entsprechende Qualifikation der Informationsdienstleister. Die menschliche Kreativität wird dabei bei der Entwicklung und Erstellung innovativer Informationsdienstleistungen der Automatisierung dieser Leistungen durch IuK-Technologie immer voraus sein. Gleichzeitig stellt aber die Substitution bestehender Informationsdienstleistungen durch Informationsprodukte auch eine Voraussetzung für die Schaffung innovativer Leistungen dar, da durch sie das notwendige Humankapital freigestellt werden kann.

\section{Implikationen für Dienstleistungsanbieter und die Rolle der Wirtschaftsinformatik}

Die zunehmende Substitution von Dienstleistungen durch Informationsprodukte im Rahmen des Internet-Handels ändert sowohl die Qualifikationsanforderungen an die Mitarbeiter als auch die strategischen Erfolgsfaktoren für Dienstleistungsunternehmen. Die Anzahl der Mitarbeiter, die im direkten Kundenkontakt einfache Informationsdienstleistungen erstellen, wird sinken. Im Gegenzug wird die Zahl derer, die mit der Entwicklung und Herstellung von Informationsprodukten befaßt sind, steigen. Von der individuellen Kundenbetreuung verschiebt sich der

11 Ein intelligenter, dosierter Einsatz von Informationsdienstleistungen kann jedoch selbst auch als Instrument zur Differenzierung benutzt werden. So bietet z.B. die Firma Virtual Vineyards (http://www.virtualvin.com), die Weine über das Internet vertreibt, als besonderen Service die individuelle Beratung von Kunden nach dessen speziellen Wünschen durch einen Weinexperten per e-mail an.

12 Inwieweit diese Vorteile tatsächlich den Kunden zugute kommen, hängt auch davon ab, wie vollständig die Substitution aus der Sicht des Kunden ist. Wenn z.B. ein Kunde einer Bank den Informationen, die er in einer persönlichen Beratung erhält, einen höheren Wert beimißt als denselben Informationen, wenn er sie sich über einen Computer beschaffen muß, so stellt das Informationsprodukt kein vollständiges Substitut dar. 
Tätigkeitsbereich hin zur grundsätzlichen Analyse der Kundenwünsche mit nachfolgendem Design der geeigneten Informationsprodukte.

Die Folge ist eine Annäherung der Produktionsmethoden und Arbeitsorganisation an die Strukturen industriell produzierender Betriebe. Die zunehmende Bedeutung einer systematischen Entwicklung von innovativen Dienstleistungen (Service Engineering bzw. Service Design) kann als erstes Indiz für diese Entwicklung aufgefaßt werden. Die konkrete Implementierung von Informationsprodukten, d.h. die systematische Entwicklung von Software (Software Engineering), wird seit vielen Jahren mit Erfolg praktiziert. Die Fähigkeit, Informationsdienstleistungen in Informationsprodukte umwandeln zu können, wird zur kritischen Kernkompetenz von Unternehmen im Informationssektor. Der Wirtschaftsinformatik kommt hierbei eine Schlüsselrolle zu. In Zusammenarbeit mit den Experten für die jeweilige Dienstleistung müssen die Möglichkeiten und Grenzen der Technik den Substitutionspotentialen gegenübergestellt werden. Grundsätzlich stehen dabei alle Arten von Informationsdienstleistungen auf dem Prüfstand. Gleichzeitig kommt dabei der Fähigkeit, menschliche Kreativität bei der Gestaltung innovativer Informationsdienstleistungen optimal einzusetzen, ebenfalls hohe Bedeutung zu. Auch hier sind Instrumente wie Service Design und Service Engineering zur Ausschöpfung dieser Potentiale nützlich.

Die vorangegangenen Überlegungen haben gezeigt, daß der Weg in die Informationsgesellschaft nicht auch zwangsläufig ein Weg in die Dienstleistungsgesellschaft ist. Informationsgüter können sowohl industriell als Informationsprodukte als auch als Informationsdienstleistungen erstellt werden. Elektronische Märkte werden dazu beitragen, daß immer mehr Informationsprodukte bestehende Informationsdienstleistungen ersetzen werden. Dabei eröffnet sich gleichzeitig die Chance für neue Informationsdienstleistungen: Die Bedeutung von Informationen als wirtschaftliches Gut wird durch beide Entwicklungen in der Zukunft noch weiter zunehmen.

\section{Literaturverzeichnis}

Anderson, C. (1997): Electronic Commerce: A Survey, The Economist, May 10 ${ }^{\text {th }}$, 1997.

Bakos, Y. (1998): The Emerging Role of Electronic Marketplaces on the Internet, in: Communications of the ACM, August 1998/Vol.41, No. 8, S.35-42.

Benjamin, R./Wigand, R. (1995): Electronic Markets and Virtual Value Chains on the Information Superhighway, in: Sloan Management Review, Winter 1995, S.62-72. 
Bode J. (1997): Der Informationsbegriff in der Betriebswirtschaftslehre, in: Schmalenbachs Zeitschrift für betriebswirtschaftliche Forschung, Heft 5, Mai 1997, S.449-468.

Choi, S.-Y./Stahl, D. O./Whinston, A. B. (1997): The Economics of Electronic Commerce, Macmillan, Indianapolis 1997.

Corsten, H. (1985): Die Produktion von Dienstleistungen, Schmidt, Berlin 1985.

Corsten, H. (1990): Betriebswirtschaftslehre der Dienstleistungsunternehmen, 2. Auflage, Oldenbourg, München 1990.

ECE (1998): Executive Research Report Electronic Commerce Enquête 1997/98, Institut für Informatik und Gesellschaft - Telematik, Universität Freiburg 1998.

Gries, W. (1995): Dienstleistungen für das 21. Jahrhundert: Chancen Nutzen Risiken bewältigen, in: Bullinger, H.-J., Dienstleistung der Zukunft Märkte, Unternehmen und Infrastrukturen im Wandel, Wiesbaden 1995, S. 3-23.

Haisken-De New, J./Horn, G.A./Schupp, J./Wagner, G. (1998): Das DienstleistungsPuzzle: Ein aktualisierter deutsch-amerikanischer Vergleich, in: DIW Wochenbericht 35/98.

Kraus, B. (1997): Arbeitsplatzeinsparungen, in: Thome, R. (Hrsg.) Arbeit ohne Zukunft?, Vahlen, München 1997.

Meyer, A. (1983) Dienstleistungsmarketing: Erkenntnisse und praktische Beispiele, 1. Auflage, Augsburg 1983.

Maleri, R. (1994): Grundlagen der Dienstleistungsproduktion, 3. Auflage, Springer, Berlin u.a. 1994.

Meffert, H./Bruhn, M. (1997): Dienstleistungsmarketing, 2. Auflage, Gabler, Wiesbaden 1997.

Nieschlag, R./Dichtl, E./Hörschgen, H. (1994): Marketing, 17. Auflage, Duncker und Humblot, Berlin 1994.

Picot, A./Reichwald, R./Wigand, R. (1998): Die grenzenlose Unternehmung, 3. Auflage, Gabler, Wiesbaden 1998.

Rayport, J.F./Sviokla, J.J. (1994): Managing in the Marketspace, in: Harvard Business Review, November-December 1994, S.141-150.

Reichwald, R./Möslein, K. (1995): Wertschöpfung und Produktivität von Dienstleistungen? - Innovationsstrategien für die Standortsicherung, in: Bullinger, H.-J., Dienstleistung der Zukunft - Märkte, Unternehmen und Infrastrukturen im Wandel, Wiesbaden 1995, S. 324-376.

Schmid, B. (1993): Elektronische Märkte, in: Wirtschaftsinformatik, 35 (1993) 5, S.465-480.

Tirole, J. (1995): Industrieökonomik, Oldenbourg, München, 1995. 
Ware, J./Gebauer, J./Hartmann, A./Roldan, M. (1998): The Search for Digital Excellence, McGraw-Hill, New York u.a. 1998. 